\title{
Replication and refinement of linkage of posterior polymorphous corneal dystrophy to the posterior polymorphous corneal dystrophy 1 locus on chromosome 20
}

Vivek S. Yellore, $P h D^{1}$, Jeanette C. Papp, $P h D^{2}$, Eric Sobel, $P h D^{2}$, M. Ali Khan, BA ${ }^{1}$, Sylvia A. Rayner, BSc ${ }^{1}$, Debora B. Farber, $P h D^{3}$, and Anthony J. Aldave, $M D^{1}$

\begin{abstract}
Purpose: The study purpose was to identify the genetic basis of posterior polymorphous corneal dystrophy, an autosomal dominant disorder of the corneal endothelium that is associated with the development of corneal edema, necessitating corneal transplantation for visual rehabilitation. Glaucoma also develops in up to $40 \%$ of patients with posterior polymorphous corneal dystrophy. Methods: Linkage analysis, using microsatellite markers previously used to demonstrate linkage of posterior polymorphous corneal dystrophy to the chromosome 20 candidate region known as posterior polymorphous corneal dystrophy 1, was performed in 29 members of a family with posterior polymorphous corneal dystrophy. Thirty-four microsatellite markers were used to refine the posterior polymorphous corneal dystrophy 1 interval. TCF8, located on chromosome 10, was screened in an affected family member to exclude posterior polymorphous corneal dystrophy 3. Results: Significant evidence of linkage to the posterior polymorphous corneal dystrophy 1 interval was obtained with both single-point and multipoint analyses. The largest single-point log odds ratio score obtained was $4.38(\theta=0)$ at marker D20S471; within 4.7 Mbp (7.2 cM) of D20S471 eight markers provided single-point log odds ratio scores of greater than 3.00 and three markers provided single-point log odds ratio scores greater than 4.00 . The largest multipoint log odds ratio score obtained was 4.83 , found across the adjacent markers D20S844, D20S191, D20S484, and D20S111. The support interval for posterior polymorphous corneal dystrophy 1 in the family we report is approximately $13.5 \mathrm{Mbp}(10 \mathrm{cM})$ long and lies between the markers D20S182 and D20S195. Eleven markers have multipoint log odds ratio scores greater than 4.0 within this region. No coding region mutations were identified in TCF8 in an affected member of the family, effectively excluding posterior polymorphous corneal dystrophy 3. Conclusions: The originally described $19.8 \mathrm{cM}$ posterior polymorphous corneal dystrophy 1 candidate disease interval has been refined to a $10 \mathrm{cM}$ interval between markers D20S182 and D20S195. A portion of this refined interval overlaps a more recently reported posterior polymorphous corneal dystrophy 1 interval, with only 20 known and predicted genes mapped to the 2.4 cM common interval. Genet Med 2007:9(4):228-234.
\end{abstract}

Key Words: Linkage analysis, PPCD, chromosome 20

Posterior polymorphous corneal dystrophy (PPCD) (Mendelian Inheritance in Man [MIM] 122000) is an autosomal dominant corneal dystrophy associated with a wide spectrum of clinical features, ranging from isolated, asymptomatic cor-

From the ${ }^{1}$ Cornea Service, The Jules Stein Eye Institute; ${ }^{2}$ Department of Human Genetics; and ${ }^{3}$ Retinal Biochemistry Laboratory, Jules Stein Eye Institute, David Geffen School of Medicine at University of California Los Angeles, California.

Anthony J. Aldave, MD, Assistant Professor, The Jules Stein Eye Institute, 100 Stein Plaza, UCLA, Los Angeles, California 90095. E-mail: aldave@jsei.ucla.edu.

The authors declare no conflict of interest.

A supplementary haplotype accompanying Figure 2 is available via the ArticlePlus feature at www.geneticsinmedicine.org. Please go to the April issue and click on the ArticlePlus link posted with the article in the Table of Contents to view this material.

Submitted for publication January 12, 2006.

Accepted for publication January 15, 2007.

DOI: 10.1097/GIM.0b013e31803c4dc2 neal endothelial morphologic changes to visually disabling corneal edema and glaucoma. Characteristic corneal endothelial abnormalities include grouped vesicles, endothelial bands, and geographic gray opacities. If the abnormal corneal endothelial cells that characterize PPCD extend across the anterior chamber angle and onto the surface of the iris, secondary angle-closure glaucoma, iridocorneal adhesions, and corectopia develop. Although the corneal dystrophies typically are not characterized by extracorneal manifestations, PPCD has also been associated with a number of ocular and systemic disorders, including keratoconus (Fig. 1).

Although the first report of an investigation into the genetic basis of PPCD was published a decade ago, ${ }^{1}$ the genetic basis of PPCD has not been firmly established in the majority of families described to date. In that original report, Heon and colleagues ${ }^{1}$ performed a genome-wide linkage analysis in a sin- 


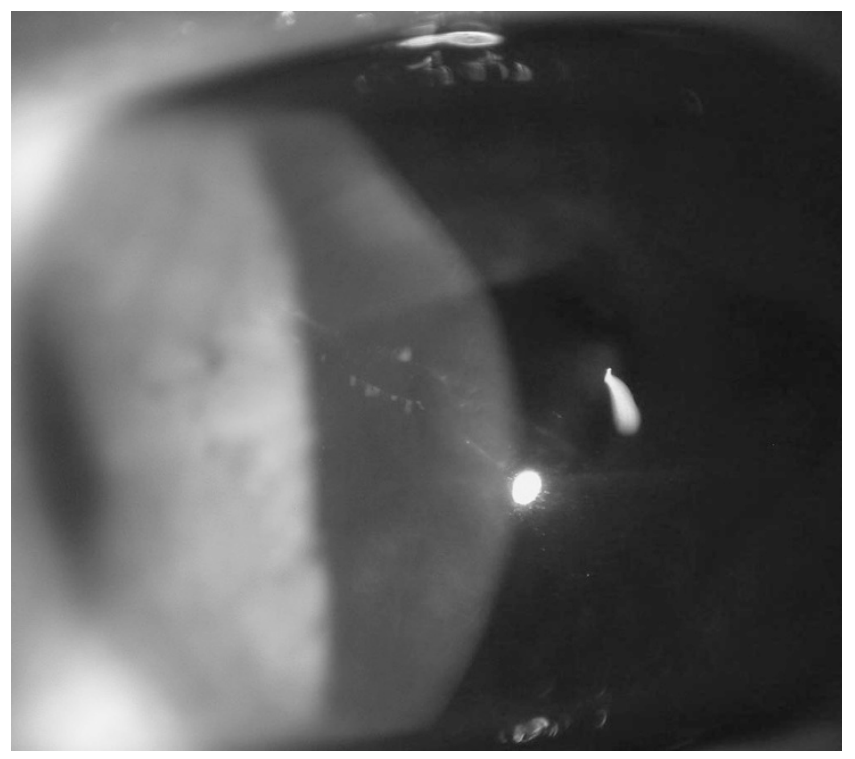

Fig. 1. Slit-lamp photomicrograph of a patient with both PPCD and keratoconus, demonstrating an endothelial band associated with PPCD and an epithelial iron line, also known as a Fleischer ring, characteristic of keratoconus. The patient is not a member of the family depicted in Figure 2, in which no individuals demonstrated features of keratoconus.

gle large pedigree with PPCD, demonstrating significant evidence of linkage to a $30 \mathrm{cM}$ (now $19.8 \mathrm{cM}$ ) pericentromeric region on the long arm of chromosome 20. In 2002, Heon and colleagues $^{2}$ reported two mutations associated with PPCD in the visual system homeobox 1 gene (VSX1, MIM 605020), which was selected for screening because it localizes to the chromosome 20 candidate gene region and is expressed in the eye. However, we have reported that the two identified sequence variants do not appear to be associated with PPCD, leading to uncertainty regarding the role of the VSX1 gene in PPCD. ${ }^{3,4}$

Gwilliam and colleagues ${ }^{5}$ also reported linkage of PPCD to the chromosome 20 candidate interval in two large Czech fam- ilies, narrowing the candidate gene region and excluding the VSX1 gene in the process. Their $2.7 \mathrm{cM}$ critical interval defined by the markers D20S48 and D20S139 overlaps the interval to which the autosomal dominant form of congenital hereditary endothelial dystrophy (CHED 1, MIM 121700) has been mapped, leading to speculation that the two conditions may be caused by different mutations in the same gene. ${ }^{6}$

Biswas and colleagues ${ }^{7}$ also reported that PPCD is associated with a mutation in the collagen, type VIII, alpha 2 gene (COL8A2, MIM 120252), located on the short arm of chromosome 1 . They chose to screen the COL8A2 gene in a patient with PPCD after a genome-wide linkage analysis in a family with early-onset Fuchs endothelial corneal dystrophy displayed linkage to a 6 - to $7 \mathrm{cM}$ region on chromosome 1 containing the COL8A2 gene. Presumed disease-causing mutations were identified in the COL8A2 gene in patients with Fuchs endothelial corneal dystrophy and 2 of 15 affected patients with PPCD. ${ }^{7}$ However, other investigators, including the current authors, have not identified any presumed pathogenic mutations in the COL8A2 gene in a large number of unrelated patients with PPCD, raising questions regarding the role of the COL8A2 gene in PPCD. ${ }^{8-10}$

A third locus for PPCD (PPCD3) was mapped to an $8.55 \mathrm{cM}$ region on chromosome 10p in a single large family by Shimizu and colleagues, ${ }^{11}$ following exclusion of the chromosome $20 \mathrm{q}$ (redesignated PPCD1) and the chromosome 1p (PPCD2) loci. Thus, with the results of this genome-wide linkage analysis in a large PPCD pedigree, genetic heterogeneity was established for this autosomal dominant corneal dystrophy. A functional evaluation of the positional candidate genes on chromosome 10 led to the selection of the TCF8 gene for screening. A frameshift mutation was found in all affected members of the family, and other frameshift and nonsense coding region mutations were identified in 4 of 10 other families screened, leading to the implication of TCF8 in the pathogenesis of PPCD. ${ }^{10}$

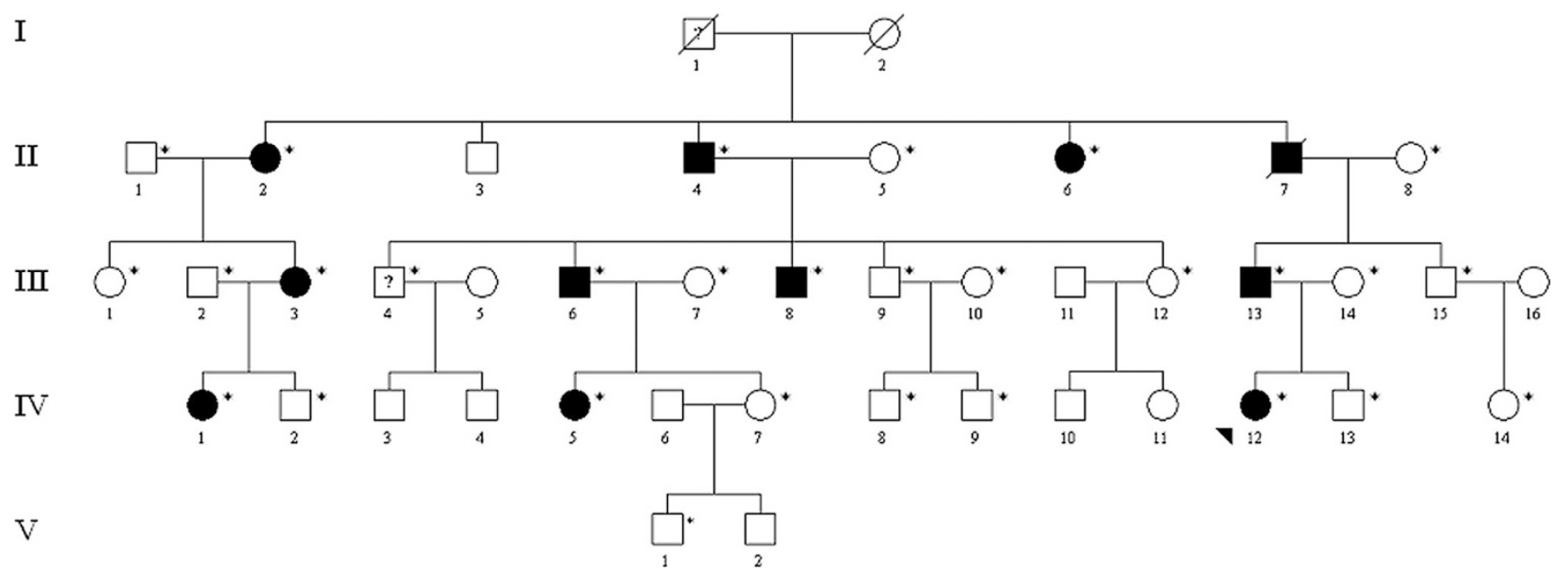

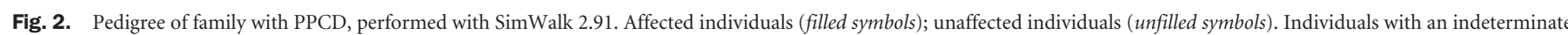

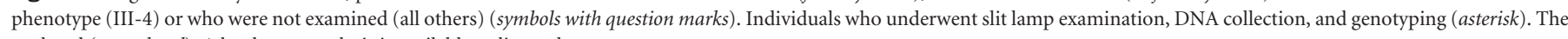
proband (arrowhead). A haplotype analysis is available online only. 
To investigate the genetic basis of PPCD in the largest of our affected families, we performed linkage analysis using microsatellite markers across the PPCD1 candidate gene region. TCF8 was screened in an affected member of the family who had undergone corneal transplantation to exclude the presence of a coding region mutation.

\section{MATERIALS AND METHODS}

The researchers followed the tenets of the Declaration of Helsinki in the treatment of the subjects reported. Study approval was obtained from the institutional review board at The University of California, Los Angeles (02-10-092-01).

\section{Patient identification/DNA collection and preparation}

After informed consent was obtained, affected patients were enrolled in the study. The diagnosis of PPCD was based on the presence of one or more of the following characteristic corneal endothelial changes in one or both eyes: scalloped bands, clustered "vesicles" with a surrounding gray halo, and geographic gray opacities. In patients who had previously undergone corneal transplantation, the diagnosis was also confirmed with histopathologic examination of the excised corneal button, when available. Unaffected family members were also enrolled in the study after informed consent was obtained, and a slitlamp examination was performed to confirm their unaffected status. Each affected and unaffected member of the family was also examined for the characteristic corneal features of keratoconus: conical corneal deformation, corneal stromal thinning, Vogt striae, and a Fleischer ring. Blood was obtained from nearly all subjects; buccal epithelial swabs were collected from children who were not cooperative with phlebotomy. Genomic DNA was prepared from the peripheral blood leukocytes and buccal epithelial cells using the Flexigene DNA Kit (Qiagen, Valencia, CA).

\section{Linkage analysis}

\section{Microsatellite markers, polymerase chain reaction amplification, and genotyping}

Thirty-four microsatellite markers spanning an $88 \mathrm{cM}$ region of chromosome 20 were selected from the National Center for Biotechnology Information (NCBI) public database, with 31 of the markers within the originally described PPCD1 interval, including the two flanking markers, D20S98 and D20S108 ${ }^{1}$ (Table 1). The locations of the markers were determined by referencing physical (NCBI human genome release, build 36.2) and genetic (Marshfield Clinic Center for Human Genetics) maps.

Polymerase chain reaction (PCR) amplification of all markers was performed using primer sequences obtained from the uniSTS section of the NCBI database. The forward primer of each pair was labeled with one of the following fluorescent dye tags: 6-FAM, VIC, or NED (Applied Biosystems, Foster City, $\mathrm{CA})$. Each reaction was carried out in a $25-\mu \mathrm{L}$ mixture containing 60 to $70 \mathrm{ng}$ of preamplified genomic DNA (amplified using GenomiPhi DNA amplification kit from Amersham Bio- sciences Corp, Piscataway, NJ), $0.25 \mu \mathrm{L}$ of each primer (10 $\mathrm{pM} / \mu \mathrm{L}), 0.5$ units Taq DNA polymerase, and $2.5 \mu \mathrm{L} 10 \times \mathrm{PCR}$ buffer (Sigma-Aldrich Co., St. Louis, MO). Thermal cycling was performed in an iCycler thermal cycler (BioRad, Hercules, $\mathrm{CA})$ under the following conditions: initial denaturation for 5 minutes at $95^{\circ} \mathrm{C} ; 30$ cycles at $94^{\circ} \mathrm{C}$ for 30 seconds, $58^{\circ} \mathrm{C}$ for 30 seconds, $72^{\circ} \mathrm{C}$ for 30 seconds; and a final extension for $5 \mathrm{~min}$ utes at $72^{\circ} \mathrm{C}$.

Genotyping was performed at the University of California at Los Angeles Sequencing and Genotyping Core. PCR fragments were analyzed on an ABI3700 and 3730 capillary DNA analyzers (Applied Biosystems). Each run included two positive control samples (individual 2 in CEPH family 1347; Coriell Institute). Genotype calling was performed using the Applied Biosystems Genotyper and Genemapper Software. All genotyping was performed blind to family structure and verified by human inspection in two independent readings.

\section{Statistical analysis}

Before linkage analysis, multipoint mistyping analysis was performed using SimWalk 2.91 to identify possible genotyping errors. ${ }^{12}$ All genotypes with greater than 0.5 posterior mistyping probability were reevaluated by review of the raw genotype data. If the possible mistyping was not resolved by review, the suspect genotypes were set to unknown.

Single-point linkage analysis was performed with Mendel $7.0,{ }^{13}$ and multipoint linkage analysis was performed with SimWalk 2.91. Linkage analysis was performed using an autosomal dominant model of inheritance, assuming a disease allele frequency of 0.001 , an incomplete penetrance of 0.9999 , and a phenocopy rate of 0.001 for both single-point and multipoint analysis. A $P$ value less than .01 was considered evidence of confirmed linkage to a region that has demonstrated significant linkage in a previous study. ${ }^{14}$ Haplotyping was performed using SimWalk 2.91.15

\section{TCF8 screening}

\section{Polymerase chain reaction amplification}

Each of the nine exons of TCF8 was amplified using the previously described primers and conditions, ${ }^{10}$ with the exception of exon 1, which was amplified using custom-designed oligonucleotide primers (forward/reverse: agcagtgcccacggttgc/ gagacataacggttcagggagagc) in a $25-\mu \mathrm{L}$ reaction that contained

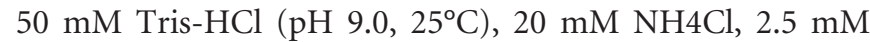
MgSO4, $200 \mu \mathrm{M}$ each dNTP plus $20 \mu \mathrm{M}$ 7-deaza-dGTP, $0.5 \mathrm{M}$ betaine, $2.5 \mu \mathrm{L}$ dimethyl sulfoxide, $150 \mathrm{mM}$ trehalose, $0.002 \%$ Tween-20, $0.12 \mu \mathrm{M}$ of each primer, 0.5 units of REDTaq Genomic DNA Polymerase (Sigma-Aldrich), and approximately $60 \mathrm{ng}$ of genomic DNA. Thermal cycling was performed in an iCycler thermal cycler (BioRad).

\section{DNA sequencing}

Purification of the PCR products was achieved by incubating 15 to 30 ng DNA with 5 units Exonuclease I and 0.5 units Shrimp Alkaline Phosphatase (USB Corp., Cleveland, OH) for 
Table 1

Single-point linkage analysis of chromosome 20 markers in family with posterior polymorphous corneal dystrophy

\begin{tabular}{|c|c|c|c|c|c|c|c|c|c|c|c|c|}
\hline \multirow[b]{2}{*}{ MARKER } & \multicolumn{2}{|c|}{ Position on chromosome 20} & \multirow[b]{2}{*}{$\begin{array}{l}\text { MAX } \\
\text { LOD }\end{array}$} & \multicolumn{9}{|c|}{ Recombination fraction } \\
\hline & $\begin{array}{l}\text { Physical } \\
\text { map (Mb) }\end{array}$ & $\begin{array}{l}\text { Genetic map } \\
\text { (Kosambi cM) }\end{array}$ & & 0 & 0.01 & 0.05 & 0.1 & 0.15 & 0.2 & 0.3 & 0.4 & 0.5 \\
\hline D20S482 & 4.45 & 12.24 & 0.34 & -1.63 & -0.63 & 0.04 & 0.26 & 0.33 & 0.34 & 0.25 & 0.13 & 0.00 \\
\hline D20S98 & 15.60 & 37.68 & 0.54 & -1.12 & -0.15 & 0.41 & 0.54 & 0.54 & 0.50 & 0.34 & 0.17 & 0.00 \\
\hline D20S114 & 17.20 & 39.28 & 2.86 & 1.51 & 2.45 & 2.86 & 2.79 & 2.58 & 2.29 & 1.59 & 0.74 & 0.00 \\
\hline D20S48 & 17.26 & 39.30 & 1.05 & -0.54 & 0.42 & 0.96 & 1.05 & 1.00 & 0.90 & 0.60 & 0.25 & 0.00 \\
\hline D20S605 & 17.67 & 39.94 & 4.17 & 4.17 & 4.11 & 3.81 & 3.43 & 3.03 & 2.61 & 1.71 & 0.76 & 0.00 \\
\hline D20S182 & 17.79 & 39.94 & 3.14 & 1.81 & 2.74 & 3.14 & 3.04 & 2.81 & 2.50 & 1.73 & 0.82 & 0.00 \\
\hline D20S471 & 19.78 & 42.68 & 4.38 & 4.38 & 4.31 & 4.00 & 3.61 & 3.20 & 2.76 & 1.84 & 0.84 & 0.00 \\
\hline D20S190 & 21.26 & 47.58 & 0.11 & 0.11 & 0.11 & 0.09 & 0.07 & 0.06 & 0.04 & 0.02 & 0.01 & 0.00 \\
\hline D20S472 & 22.09 & 47.58 & 4.27 & 4.27 & 4.20 & 3.90 & 3.50 & 3.08 & 2.65 & 1.72 & 0.76 & 0.00 \\
\hline D20S871 & 23.28 & 48.66 & 3.30 & 3.30 & 3.25 & 3.01 & 2.69 & 2.36 & 2.02 & 1.31 & 0.60 & 0.00 \\
\hline D20S101 & 23.59 & 48.66 & 3.19 & 3.19 & 3.14 & 2.91 & 2.62 & 2.31 & 1.99 & 1.31 & 0.58 & 0.00 \\
\hline D20S844 & 24.40 & 49.80 & 4.34 & 4.34 & 4.27 & 3.97 & 3.57 & 3.16 & 2.73 & 1.80 & 0.82 & 0.00 \\
\hline D20S191 & 26.12 & 49.80 & 1.22 & 1.22 & 1.20 & 1.10 & 0.96 & 0.81 & 0.66 & 0.36 & 0.11 & 0.00 \\
\hline D20S484 & 29.53 & 49.80 & 3.05 & 3.05 & 3.00 & 2.78 & 2.50 & 2.21 & 1.90 & 1.24 & 0.58 & 0.00 \\
\hline D20S111 & 29.94 & 49.80 & 3.65 & 3.65 & 3.58 & 3.30 & 2.93 & 2.55 & 2.16 & 1.37 & 0.58 & 0.00 \\
\hline D20S200 & 30.43 & 50.34 & 2.68 & 2.68 & 2.64 & 2.44 & 2.17 & 1.90 & 1.61 & 1.02 & 0.45 & 0.00 \\
\hline D20S843 & 30.64 & 50.34 & 2.46 & 2.46 & 2.42 & 2.22 & 1.97 & 1.72 & 1.46 & 0.93 & 0.42 & 0.00 \\
\hline D20S195 & 31.29 & 50.88 & 0.08 & -3.74 & -1.84 & -0.59 & -0.16 & 0.01 & 0.08 & 0.07 & 0.01 & 0.00 \\
\hline D20S890 & 31.60 & 50.88 & 2.16 & 2.16 & 2.12 & 1.94 & 1.71 & 1.48 & 1.24 & 0.75 & 0.30 & 0.00 \\
\hline D20S878 & 31.93 & 50.88 & 0.07 & -1.76 & -0.78 & -0.18 & 0.00 & 0.06 & 0.07 & 0.05 & 0.03 & 0.00 \\
\hline D20S106 & 32.97 & 50.88 & 2.23 & 0.85 & 1.79 & 2.23 & 2.20 & 2.03 & 1.79 & 1.20 & 0.53 & 0.00 \\
\hline D20S909 & 33.92 & 50.88 & 0.92 & -0.69 & 0.27 & 0.82 & 0.92 & 0.90 & 0.83 & 0.60 & 0.32 & 0.00 \\
\hline D20S896 & 34.16 & 50.88 & 1.48 & -0.03 & 0.93 & 1.43 & 1.48 & 1.40 & 1.26 & 0.91 & 0.48 & 0.00 \\
\hline D20S847 & 34.32 & 50.88 & 2.00 & 0.54 & 1.49 & 1.98 & 2.00 & 1.87 & 1.67 & 1.12 & 0.47 & 0.00 \\
\hline D20S884 & 35.50 & 51.42 & 2.71 & 1.36 & 2.29 & 2.71 & 2.63 & 2.42 & 2.14 & 1.46 & 0.68 & 0.00 \\
\hline D20S859 & 35.88 & 51.42 & 2.02 & 0.63 & 1.57 & 2.02 & 1.99 & 1.83 & 1.61 & 1.06 & 0.48 & 0.00 \\
\hline D20S478 & 36.67 & 54.14 & 0.71 & -0.98 & -0.04 & 0.55 & 0.69 & 0.71 & 0.66 & 0.46 & 0.20 & 0.00 \\
\hline D20S174 & 37.16 & 54.14 & 3.29 & 3.29 & 3.24 & 3.00 & 2.70 & 2.38 & 2.04 & 1.34 & 0.59 & 0.00 \\
\hline D20S908 & 37.25 & 54.14 & 1.77 & 1.77 & 1.74 & 1.61 & 1.45 & 1.29 & 1.13 & 0.78 & 0.41 & 0.00 \\
\hline D20S107 & 38.32 & 55.82 & 1.93 & 0.52 & 1.47 & 1.93 & 1.92 & 1.77 & 1.56 & 1.02 & 0.43 & 0.00 \\
\hline D20S170 & 39.78 & 56.90 & 1.77 & -1.44 & 0.41 & 1.53 & 1.77 & 1.76 & 1.62 & 1.16 & 0.57 & 0.00 \\
\hline D20S108 & 40.26 & 57.44 & 0.00 & -2.88 & -1.92 & -1.21 & -0.85 & -0.62 & -0.45 & -0.23 & -0.09 & 0.00 \\
\hline D20S836 & 44.37 & 64.60 & 0.12 & -4.55 & -2.68 & -1.14 & -0.50 & -0.18 & -0.01 & 0.12 & 0.10 & 0.00 \\
\hline D20S196 & 49.00 & 75.30 & 0.00 & -4.40 & -2.42 & -1.10 & -0.60 & -0.36 & -0.23 & -0.11 & -0.06 & 0.00 \\
\hline
\end{tabular}

LOD, $\log$ odds ratio.

15 minutes at $37^{\circ} \mathrm{C}$. After inactivation of the nucleases $(15$ minutes at $80^{\circ} \mathrm{C}$ ), sequencing reactions were performed by the addition of $2 \mu \mathrm{L}$ of BigDye Terminator Mix v3.1 (Applied Biosystems), $2 \mu \mathrm{L}$ of SeqSaver (Sigma-Aldrich), and $0.2 \mu \mathrm{L}$ primer $(10 \mathrm{pM} / \mu \mathrm{L})$. Samples were denatured at $96^{\circ} \mathrm{C}$ for 2 minutes, then cycled 25 times at $96^{\circ} \mathrm{C}$ for 10 seconds, $50^{\circ} \mathrm{C}$ for 5 seconds, and $60^{\circ} \mathrm{C}$ for 4 minutes. Unincorporated nucleotides were removed using the CleanSeq reagent and an SPRI plate (Agencourt Bioscience Corporation, Beverly, MA), following the manufacturer's instructions, and then analyzed on an ABI-3100 Genetic 
Analyzer (Applied Biosystems) after resuspension in $0.1 \mathrm{mM}$ of EDTA. Nucleotide sequences, read manually and with Mutation Surveyor v2.2 (Softgenetics, State College, PA), were compared with the published TCF8 cDNA sequence (GenBank accession number NM_030751).

\section{RESULTS}

\section{Clinical findings}

Twenty-nine individuals from a large white American family with PPCD were enrolled in the study and underwent a slit-lamp examination and DNA collection. Ten individuals in three consecutive generations were classified as affected, 18 individuals had an unremarkable ocular examination and were classified as unaffected, and one individual (III-4) with an isolated corneal endothelial opacity that was not characteristic of PPCD was classified with an indeterminate phenotype. (Fig. 2) Four of the affected individuals had undergone corneal transplantation for visually significant corneal edema (II-2, II-4, II-7, III-6), one of whom (II-4) demonstrated PPCD-associated corectopia, iridocorneal adhesions, and secondary angleclosure glaucoma in one eye, and absolute glaucoma in the other. The other six affected individuals demonstrated asymptomatic involvement, with clinical features ranging from a few isolated endothelial vesicles to densely distributed endothelial vesicles and bands associated with mild corneal stromal edema. None of the enrolled family members demonstrated any of the characteristic clinical features of keratoconus.

\section{Linkage analysis}

Analysis of genotyping results yielded a largest single-point $\log$ odds ratio (LOD) score of $4.38(\theta=0)$, obtained with marker D20S471. Nine of the other 33 markers also provided single-point LOD scores greater than 3.00, eight of which are within $4.7 \mathrm{Mbp}(7.2 \mathrm{cM})$ of D20S471: D20S605 $(4.17 ; \theta=$ $0.00), \mathrm{D} 20 \mathrm{~S} 182(3.14 ; \theta=0.05), \mathrm{D} 20 \mathrm{~S} 472(4.27 ; \theta=0.00)$, D20S871 (3.30; $\theta=0.00)$, D20S101 (3.19; $\theta=0.00)$, D20S844 $(4.34 ; \theta=0.00), \mathrm{D} 20 \mathrm{~S} 484(3.05 ; \theta=0.00)$, and D20S111 (3.65; $\theta=0.00$ ) (Table 1$)$. The largest multipoint LOD score was 4.83, obtained with the four adjacent markers D20S844, D20S191, D20S484, and D20S111, which are located approximately $7 \mathrm{cM}$ from the marker D20S471. The support interval, defined as the region in which the multipoint LOD score is within 1 unit of the peak value, is approximately $13.5 \mathrm{Mbp}$ (10 cM) long and lies between markers D20S182 and D20S195 (Fig. 3). The haplotype analysis, shown in Figure 2, indicates a region conserved among all affected individuals and absent in all individuals known to be unaffected with PPCD. The conserved haplotype is also present in individual III-4, the previously mentioned individual of unknown status.

\section{TCF8 gene screening}

No coding region sequence variants were identified in an affected member of the family (II-2) who required a penetrating keratoplasty for corneal edema secondary to PPCD.

\section{DISCUSSION}

This report provides confirmation of the findings of Heon et al. ${ }^{1}$ and Gwilliam et al. ${ }^{5}$ that a locus for PPCD is present on chromosome 20. The marker that provided the highest singlepoint LOD score, D20S471, is located less than $190 \mathrm{Kbp}$ from the marker that provided the maximum LOD score in one of

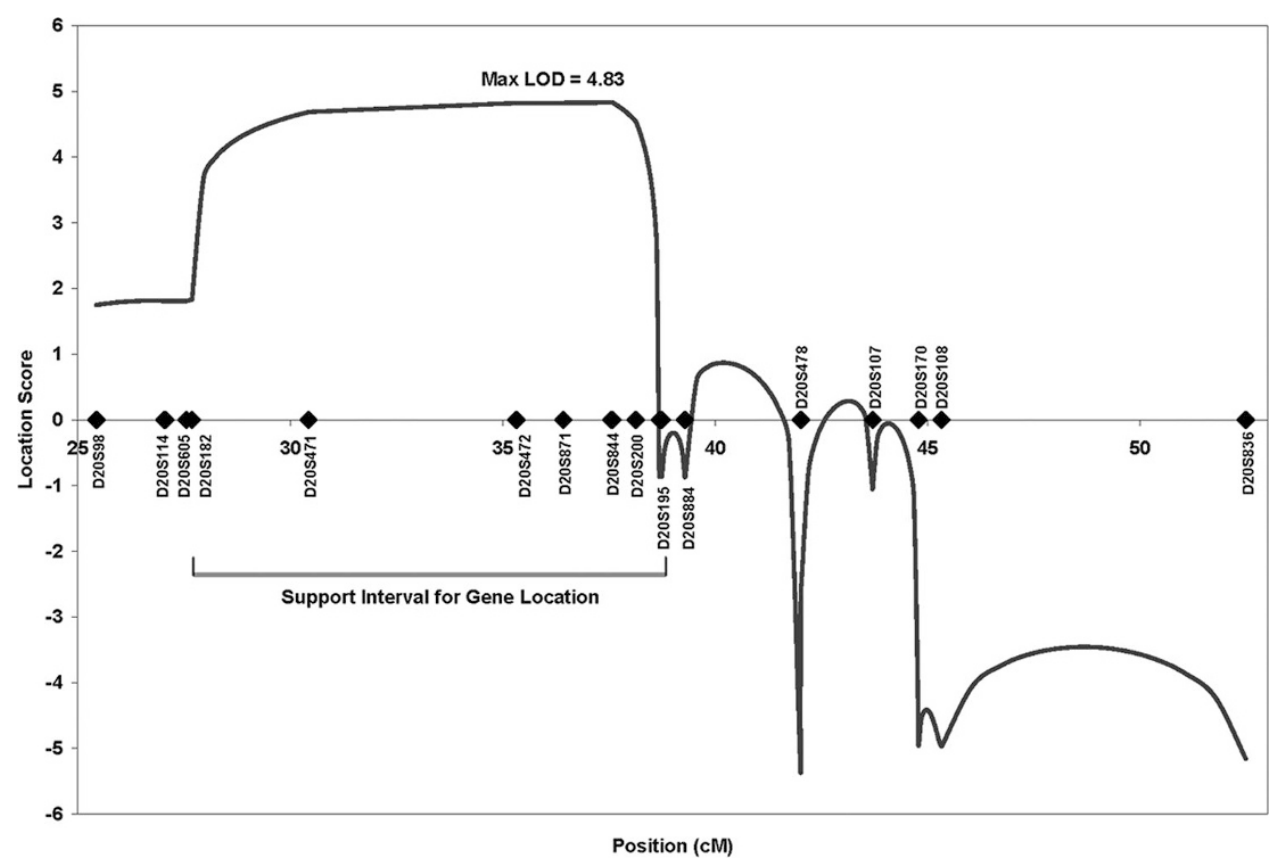

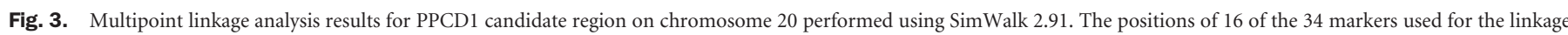

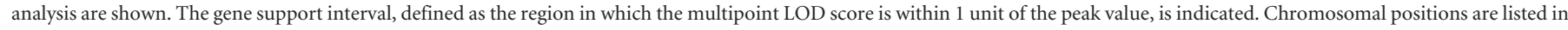
Kosambi cM. LOD, log odds ratio. 


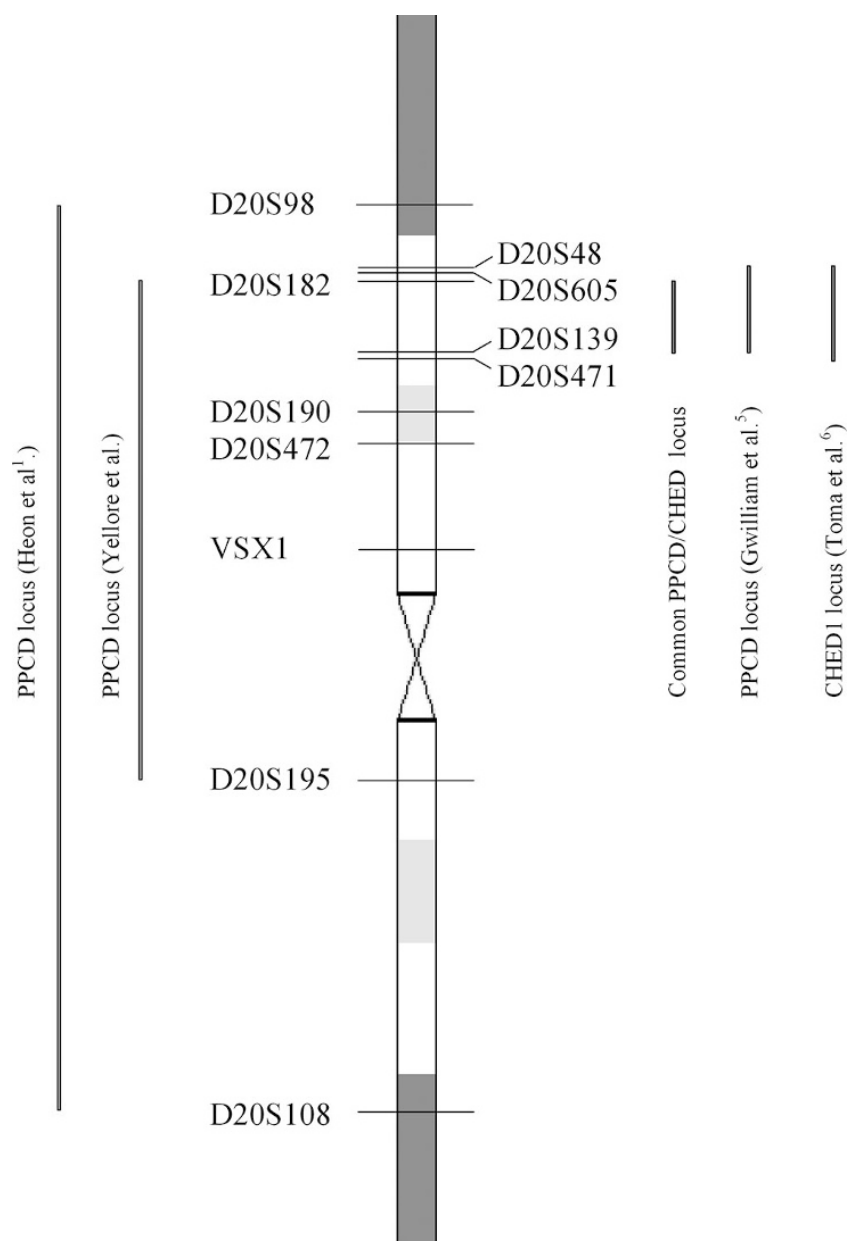

Fig. 4. Ideogram of pericentromeric portion of chromosome 20 demonstrating the original PPCD1 candidate gene interval described by Heon et al., ${ }^{1}$ bordered by markers D20S98 and D20S108; the refined candidate interval that we report, as defined by flanking markers D20S182 and D20S195; the refined interval bordered by D20S48 and D20S139 reported by Gwilliam and colleagues; ${ }^{5}$ and the CHED1 interval, defined by the markers D20S48 and D20S471. ${ }^{6}$ The PPCD1 candidate interval that is common to each of the three studies is defined by the markers D20S182 and D20S139. The VSX1 gene is located outside of this common interval. PPCD, posterior polymorphous corneal dystrophy; CHED, congenital hereditary endothelial dystrophy.

the two Czech PPCD families reported by Gwilliam et al., D20S139 (Fig. 4). The maximum LOD score obtained in the other Czech family was 3.22 with marker D20S605, which also obtained a significant single-point LOD score (4.17) in the family we report. In addition, the refined loci that Gwilliam et al. and we report are both located within the original PPCD1 interval described by Heon et al. ${ }^{1}$ (Fig. 4). Given these results, it is reasonable to conclude that the genetic cause of PPCD in the family we report is the same as in the two Czech families described by Gwilliam et al., and possibly the large family described by Heon and colleagues. ${ }^{1}$

By assuming that the genetic basis of PPCD1 is the same in the family described by Heon et al., ${ }^{1}$ the two families described by Gwilliam et al., ${ }^{5}$ and the family we report, the gene is most likely in the intersection of the support intervals from these three studies. Because only 20 genes have been mapped to this $2.4 \mathrm{cM}$ common interval, defined by the markers D20S182 and
D20S139 (www.ncbi.nlm.nih.gov; build 36.2), screening of each positional candidate gene is currently under way in our laboratory.

The identification of the genetic basis of PPCD1 will also likely result in elucidation of the genetic basis of CHED1, which has been mapped to the overlapping interval defined by the flanking markers D20S48 and D20S4716 (Fig. 4). Because the $2.7 \mathrm{cM}$ PPCD1 candidate interval reported by Gwilliam and colleagues ${ }^{5}$ is defined by the flanking markers D20S48 and D20S139, and D20S471 and D20S139 are separated by less than $190 \mathrm{Kbp}, \mathrm{PPCD} 1$ and CHED1 have been linked to essentially the identical $2.7 \mathrm{cM}$ region. It is likely, therefore, that these two dominantly inherited corneal endothelial dystrophies are caused by different mutations in the same gene, or that CHED1 may simply represent a severe, early-onset phenotypic variant of PPCD.

Although locus heterogeneity has been demonstrated for PPCD, we believe that the majority of families with multiple affected individuals will demonstrate mutations in a gene in the PPCD1 candidate region. Including this report, four of the five families with PPCD in whom linkage analysis has been performed have demonstrated linkage to the PPCD1 locus. ${ }^{1,5}$ Because linkage analysis is typically performed in large families, this localization may not hold true for PPCD that is identified in families with only a single or a few affected individuals. Krafchak and colleagues ${ }^{10}$ reported TCF 8 mutations in 5 of 11 families with PPCD who were screened, with the only mutation identified in a family with more than three affected individuals occurring near the end of the coding region of the gene. Two of the other mutations in TCF8 were identified in families with a single affected offspring of unaffected parents, and were thus assumed to be de novo mutations. ${ }^{10}$ Therefore, it is possible that the locus heterogeneity that exists for PPCD will be reflected by the presence of mutations in a gene in the PPCD1 candidate region in families with multiple affected individuals and TCF8 mutations in isolated cases of PPCD.

\section{ACKNOWLEDGMENTS}

Support was provided by the National Institutes of Health K08 EY016079, Emily Plumb Estate and Trust, William Dickerson Estate (A. J. A.), and National Institutes of Health R01 EY008285 (D. B. F.). We thank Dr. Geoffrey C. Tabin for contributing patients to this project and Ram Sripracha for his programming expertise and assistance in drawing the PPCD family haplotypes.

\section{References}

1. Heon E, Mathers WD, Alward WL, Weisenthal RW, et al. Linkage of posterior polymorphous corneal dystrophy to 20q11. Hum Mol Genet 1995;4(3):485-488.

2. Heon E, Greenberg A, Kopp KK, Rootman D, et al. VSX1: a gene for posterior polymorphous dystrophy and keratoconus. Hum Mol Genet 2002;11(9):1029-1036.

3. Aldave AJ, Yellore VS, Principe AH, Abedi G, et al. Candidate gene screening for posterior polymorphous dystrophy. Cornea 2005;24(2):151-155.

4. Aldave AJ, Sonmez B. Elucidating the molecular genetic basis of the corneal dystrophies: are we there yet? Arch Ophthalmol 2007;125:1-10.

5. Gwilliam R, Liskova P, Filipec M, Kmoch S, et al. Posterior polymorphous corneal dystrophy in Czech families maps to chromosome 20 and excludes the VSX1 gene. Invest Ophthalmol Vis Sci 2005;46(12):4480-4484. 
6. Toma NM, Ebenezer ND, Inglehearn CF, Plant C, et al. Linkage of congenital hereditary endothelial dystrophy to chromosome 20. Hum Mol Genet 1995;4(12): 2395-2398.

7. Biswas S, Munier FL, Yardley J, Hart-Holden N, et al. Missense mutations in COL8A2, the gene encoding the alpha2 chain of type VIII collagen, cause two forms of corneal endothelial dystrophy. Hum Mol Genet 2001;10(21):24152423.

8. Kobayashi A, Fujiki K, Murakami A, Kato T, et al. Analysis of COL8A2 gene mutation in Japanese patients with Fuchs' endothelial dystrophy and posterior polymorphous dystrophy. Jpn J Ophthalmol 2004;48(3):195-198.

9. Yellore VS, Rayner SA, Emmert-Buck L, Tabin GC, et al. No pathogenic mutations identified in the COL8A2 gene or four positional candidate genes in patients with posterior polymorphous corneal dystrophy. Invest Ophthalmol Vis Sci 2005;46(5): 1599-1603.
10. Krafchak CM, Pawar H, Moroi SE, Sugar A, et al. Mutations in TCF8 cause posterior polymorphous corneal dystrophy and ectopic expression of COL4A3 by corneal endothelial cells. Am J Hum Genet 2005;77:694-708.

11. Shimizu S, Krafchak C, Fuse N, Epstein MP, et al. A locus for posterior polymorphous corneal dystrophy (PPCD3) maps to chromosome 10. Am J Med Genet A 2004;130:372-377.

12. Sobel E, Lange K. Descent graphs in pedigree analysis: applications to haplotyping location scores, and marker-sharing statistics. Am J Hum Genet 1996;58(6):1323-1337.

13. Lange K, Sinsheimer JS, Sobel E. Association testing with Mendel. Genet Epidemiol 2005;29(1):36-50.

14. Lander E, Kruglyak L. Genetic dissection of complex traits: guidelines for interpreting and reporting linkage results. Nat Genet 1995;11(3):241-247.

15. Sobel E, Papp JC, Lange K. Detection and integration of genotyping errors in statistical genetics. Am J Hum Genet 2002;70(2):496-508. 